\title{
Two-crystal mid-infrared optical parametric oscillator for absorption and dispersion dual-comb spectroscopy
}

\author{
Yuwei Jin, Simona M. Cristescu, Frans J. M. Harren, and Julien Mandon* \\ Molecular and Laser Physics, Institute for Molecular and Materials, Radboud University, P.O. Box 9010, 6500 GL Nijmegen, The Netherlands \\ *Corresponding author: j.mandon@science.ru.nl
}

Received March 21, 2014; revised April 14, 2014; accepted April 21, 2014; posted April 25, 2014 (Doc. ID 208686); published May 27, 2014

\begin{abstract}
We present a femtosecond optical parametric oscillator (OPO) containing two magnesium-doped periodically poled lithium niobate crystals in a singly resonant ring cavity, pumped by two mode-locked Yb-fiber lasers. As such, the OPO generates two idler combs (up to $220 \mathrm{~mW}$ ), covering a wavelength range from 2.7 to $4.2 \mu \mathrm{m}$, from which a midinfrared dual-comb Fourier transform spectrometer is constructed. By detecting the heterodyning signal between the two idler beams a full broadband spectrum of a molecular gas can be observed over $250 \mathrm{~cm}^{-1}$ within $70 \mu \mathrm{s}$ with a spectral resolution of $15 \mathrm{GHz}$. The absorption and dispersion spectra of acetylene and methane have been measured around $3000 \mathrm{~cm}^{-1}$, indicating that this OPO represents an ideal broadband mid-infrared source for fast chemical sensing. (C) 2014 Optical Society of America

OCIS codes: (300.6300) Spectroscopy, Fourier transforms; (300.6390) Spectroscopy, molecular; (120.6200) Spectrometers and spectroscopic instrumentation; (190.4970) Parametric oscillators and amplifiers.

http://dx.doi.org/10.1364/OL.39.003270
\end{abstract}

In the mid-infrared wavelength region between 2 and $20 \mu \mathrm{m}$, gases, liquids, and solids have unique absorption and dispersion features that are associated with their molecular rotational-vibrational transitions. To observe these spectra, a wide variety of instrumentations is available for quantitative analysis, such as Fourier transform spectrometry (FTS). With the recent development of laser frequency combs, a novel approach can be used for measuring the broadband spectra through multiheterodyne spectroscopy [ $[1]$.

To date, commercially available frequency combs are limited to the near-infrared region up to $2 \mu \mathrm{m}$. Therefore, spectrometers based on frequency combs offer limited sensitivity, as the molecular transitions have typically two orders of magnitude weaker absorption cross sections in the near-infrared wavelength region as compared to the mid-infrared. This is why research on mid-infrared frequency comb sources is of great interest. Four strategies can be considered: the use of mode-locked lasers with a gain medium producing direct mid-infrared radiation, different frequency generation (DFG), optical parametric oscillation, or Kerr comb generation [2]. Among those, the optical parametric oscillator (OPO) offers a wide tuning range in the mid-infrared region and high conversion efficiency. In the past, OPOs pumped by Ti:sapphire femtosecond lasers were demonstrated as efficient sources for broadband and sensitive spectroscopy $[3,4]$. Later on, fully stabilized OPO-based combs have been demonstrated for wavelengths longer than $2.5 \mu \mathrm{m}$, either via singly resonant OPOs $[5,6]$ or double-resonant OPOs $[\underline{7}, 8]$ generating ultrabroad mid-infrared spectra.

Different approaches have been developed to use frequency combs systems as light sources for absorption spectroscopy. Taking advantage of the wide spectral emission, near-infrared molecular spectra were recorded with multichannel arrangements [9-11], multiplex spectrometers $[12,13]$ or different types of dual-comb spectrometry [1,14-19]. In combination with a multipass gas cell or a high finesse optical cavity, it is possible to achieve high sensitivity to trace molecular gases at part per billion levels [1,9,10,20,21].

The development of dual-comb FTS overcomes several disadvantages of conventional FTS $[\underline{14}, \underline{22}]$ and gives simultaneous measurements of the absorption and dispersion spectra of a sample. Several approaches have been demonstrated to perform mid-infrared dual-comb spectroscopy $[16,18,23]$. Among those, DFG-based systems have provided accurate spectral measurements around $3.4 \mu \mathrm{m}$ [16]. However, microwatt power levels and the relative narrow spectral bandwidth of the DFG source can limit its implementation in applications such as real-time multigas detection.

Here, we report on a high-power mid-infrared OPO containing two MgO-doped periodically poled lithium niobate (MgO:PPLN) crystals in a singly resonant ring cavity, pumped by two mode-locked lasers. If the idea of two-crystal OPOs has been demonstrated for the generation of continuous wave near-infrared light [24], for the first time it is implemented on mid-infrared frequency combs. The special and unique design of the OPO cavity offers several remarkable advantages for dual-comb spectroscopy, such as the emission of two independent mid-infrared beams separated in space and a significant average optical power of $220 \mathrm{~mW}$.

The configuration of the OPO is depicted in Fig. 1. The pump lasers (Menlo Systems, Germany) are femtosecond $\mathrm{Yb}$-fiber lasers, emitting at a central wavelength of $1040 \mathrm{~nm}\left(9615 \mathrm{~cm}^{-1}\right)$, delivering up to $2 \mathrm{~W}$ of average power with $80 \mathrm{fs}$ optical pulse duration $(30 \mathrm{~nm}$ or $275 \mathrm{~cm}^{-1}$ spectral width at $1 / e$ value); the collimated beams have diameters of 2.4 and $2.8 \mathrm{~mm}$. The repetition frequency of one pump laser (90 MHz) is stabilized by synchronizing its 10th harmonic to a frequency generator (R\&S, SMB100A) offering a single sideband phase noise of $-128 \mathrm{dBc}$ at $1 \mathrm{GHz}$. The 10th harmonic of the repetition rate of the second pump laser is locked to the same reference clock with a slightly different frequency of up to $3 \mathrm{kHz}$, corresponding to a $300 \mathrm{~Hz}$ different repetition 


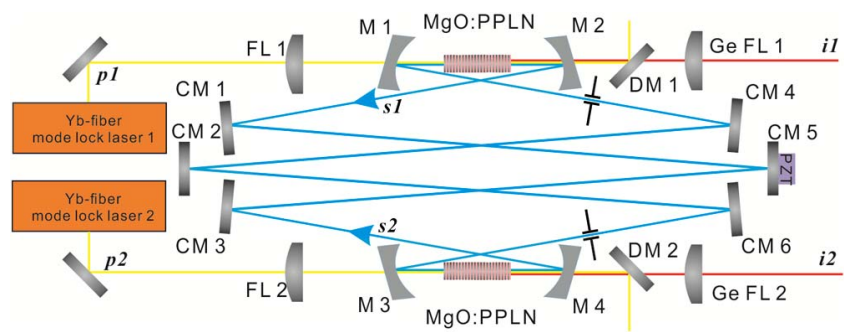

Fig. 1. Experimental setup of the OPO cavity. p1-2, s1-2, i1-2, pumps, signals, and idler beams, respectively, corresponding to different pump lasers. FL1-2, focal lenses $(f=100 \mathrm{~mm})$. M1-M4, curved mirrors ( $r=100 \mathrm{~mm}$ ). CM1-CM6, chirped mirrors (100 $\mathrm{fs}^{2}$ at $\left.1500 \mathrm{~nm}\right)$. DM1-2, dichroic mirrors. GeFL1-2, germanium focal lenses $(f=100 \mathrm{~mm})$. PZT, piezo actuator.

frequency. A higher difference in between the repetition frequencies will not give simultaneous oscillation within the OPO cavity for both beams. The pump beams are focused onto the OPO crystals inside the ring cavity with two lenses $(f=100 \mathrm{~mm})$. The $5 \mathrm{~mm}$ long MgO:PPLN crystals (Covesion, UK) are temperature stabilized and have eight poling periods each, ranging from 27.91 to $31.59 \mu \mathrm{m}$. Both end facets of each PPLN crystal are antireflective (AR) coated $(R<1.5 \%$ at $1064 \mathrm{~nm}, R<1 \%$ at $1400-1800 \mathrm{~nm}$, and $R<6 \%$ at $2600-4800 \mathrm{~nm}$ ). The OPO cavity is designed to be synchronously pumped; therefore, the cavity length is about $3.4 \mathrm{~m}$ long. The ring cavity consists of four curved mirrors (radius of curvature $100 \mathrm{~mm}$, AR coated at 1064 and 3650-4850 nm and high-reflective [HR] coated at 1350-1500 nm) and six flat, chirped mirrors (HR coated at 1375-1750 nm, Layertec GmbH, Germany). The chirped mirrors (CM1-CM6) have a second-order dispersion of approximately $-100 \mathrm{fs}^{2}$ per bounce at $1500 \mathrm{~nm}$. They compensate the group velocity dispersion introduced by the PPLN crystals and the curved mirrors at $1.5 \mu \mathrm{m}$. A piezo actuator is mounted on CM5 for further frequency combs stabilization. The idler beams are emitting collinearly with the residual pump beams, and two dichroic mirrors (LaserOptik $\mathrm{GmbH}$, $R>99.9 \%$ at $1064 \mathrm{~nm}$ and $R<1 \%$ at $3200-3900 \mathrm{~nm}$ ) are used to separate the pump and idler beams. Since a wide variety of non-phase-matched light beams are generated from the PPLN crystals, two germanium lenses $(f=100 \mathrm{~mm}$, AR coated at 3-5 $\mu \mathrm{m}$, Edmund Optics Ltd, UK) are filtering out and collimating the idler beams. To impede higher order transversal modes in the OPO cavity, two irises are placed. The aperture of the irises causes significant diffraction losses for the higher-order modes. This is beneficial for heterodyne detection since the two output idler beams should have an optimal spatial overlap, which can be achieved when both beams are in $\mathrm{TEM}_{00}$ mode [25].

The spectral properties of the OPO are analyzed by a rapid-scan Fourier transform spectrometer (Magna-IR E.S.P system). For this experiment, the OPO cavity is pumped by only one mode-locked laser. The spectra of the signal beam for the eight poling periods of the crystal are depicted in Fig. 2(a) (spectral resolution of $0.4 \mathrm{~cm}^{-1}$ ), ranging from 4800 to $7200 \mathrm{~cm}^{-1}(1.3-2.2 \mu \mathrm{m})$. The idler spectra are depicted in Fig. 2(b). As can be seen from the figure, a spectral range extending from 2300 to $3600 \mathrm{~cm}^{-1}$ $(2.7-4.4 \mu \mathrm{m})$ can be covered. The maximum spectral

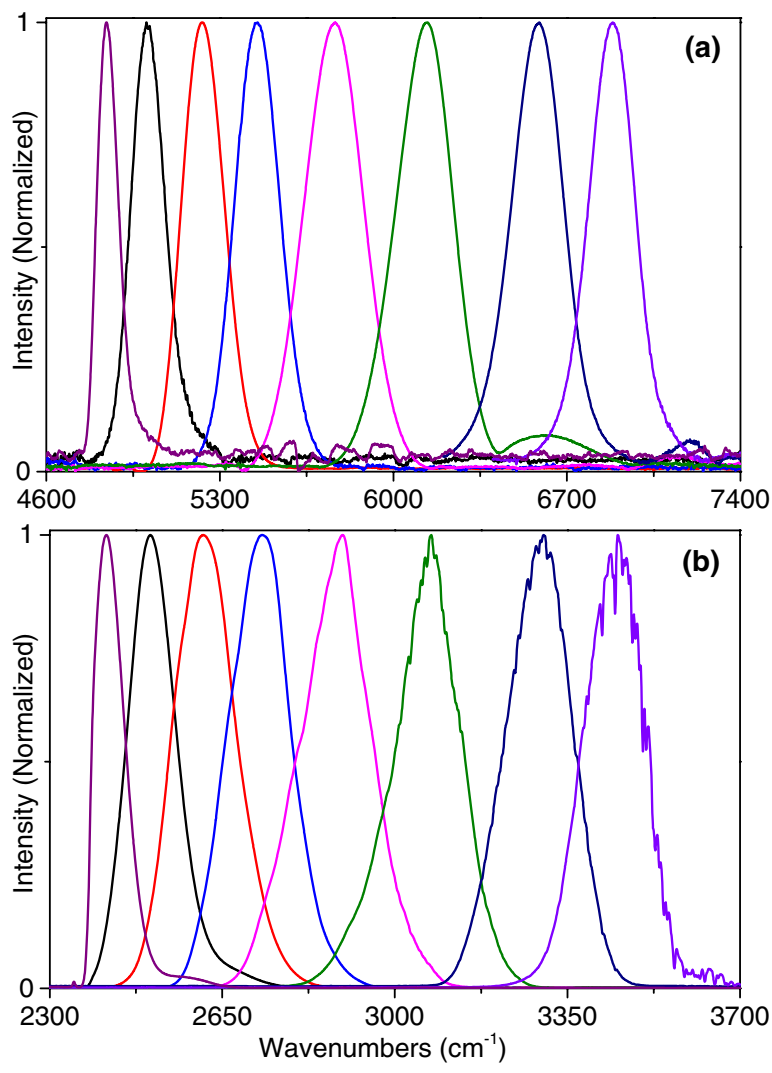

Fig. 2. (a) Spectral intensity distribution of the signal using eight poling periods, 27.91 to $31.59 \mu \mathrm{m}$, covering a wavelength region between 4800 and $7200 \mathrm{~cm}^{-1}$. (b) Spectral intensity distribution of the idler light using eight poling periods, resulting in a wavelength coverage between 2400 and $3600 \mathrm{~cm}^{-1}$.

width within one period is $300 \mathrm{~cm}^{-1}$ (1/e value), centered at $3080 \mathrm{~cm}^{-1}(3.24 \mu \mathrm{m})$. To characterize the threshold and conversion efficiency, the idler power has been measured at different crystal periods for different pump powers (see Fig. 3). A pump power threshold as low as $46 \mathrm{~mW}$ has been achieved for the poling period generating an idler spectra centered at $3300 \mathrm{~cm}^{-1}$ with a maximum conversion efficiency of $27 \%$.

To conduct spectral analysis of gas phase samples, a dual-comb FTS is constructed, which is shown in Fig. $\underline{4}$.

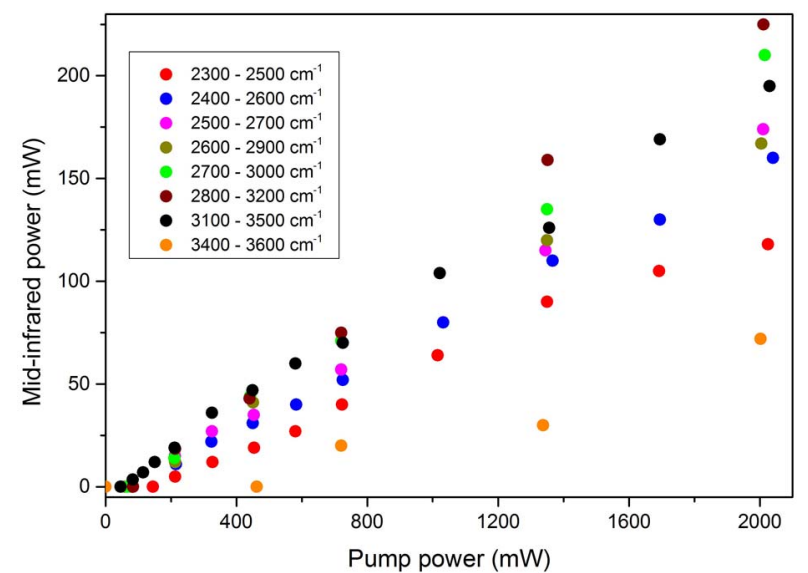

Fig. 3. Average output power of the idler at the eight different crystal periods. 


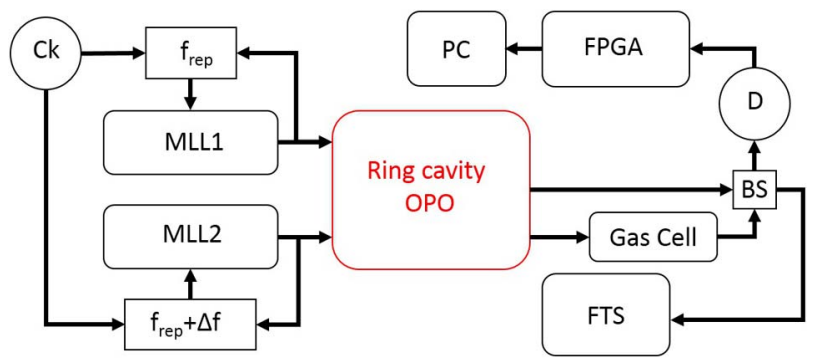

Fig. 4. Experimental setup of the dual-comb FTS. Two Ybfiber mode-locked lasers (MLL1-2) are coupled to a singly resonant ring cavity OPO. Synchronized to a reference clock (Ck), the difference between the repetition frequencies is $\Delta f$. The OPO generates two spatially separated idler beams. One idler beam probes the gas sample in a $30 \mathrm{~cm}$ long single pass cell (Gas Cell) and is recombined with the second idler beam at the beam splitter (BS). Both overlapped beams are focused and the beating signal is detected by a Peltier cooled fast IR detector (D). The FFT of the detector signal is calculated by a FPGA, giving a spectral image of the gas sample. The light from the other side of the beam splitter is analyzed by a commercial rapid-scan FTS.

It consists of two femtosecond Yb-fiber lasers, each pumping the singly resonant OPO cavity described previously. The repetition rates of both pump lasers are synchronized to the same frequency generator. The difference in repetition rate $(\Delta f)$ between the two lasers is set to values ranging from 40 to $120 \mathrm{~Hz}$ for various experiments. One collimated idler beam is probing the gas sample before interfering with the other idler beam on a single detector (Vigo, PVI-4TE, $50 \mathrm{MHz}$ bandwidth). From this fast preamplified Peltier cooled mid-infrared photodetector, the interferogram is low-pass filtered (up to $50 \mathrm{MHz}$ ) and sampled at a frequency of $125 \mathrm{MHz}$. A fast Fourier transform (FFT) of the recorded signal, calculated with a field programmable gate array (FPGA)based data acquisition system (National Instruments Netherland BV) gives a real-time downconverted image of the molecular spectra in the RF frequency domain.

Figure 5 gives an illustration of the truncated interferogram from the full recording and the calculated spectra

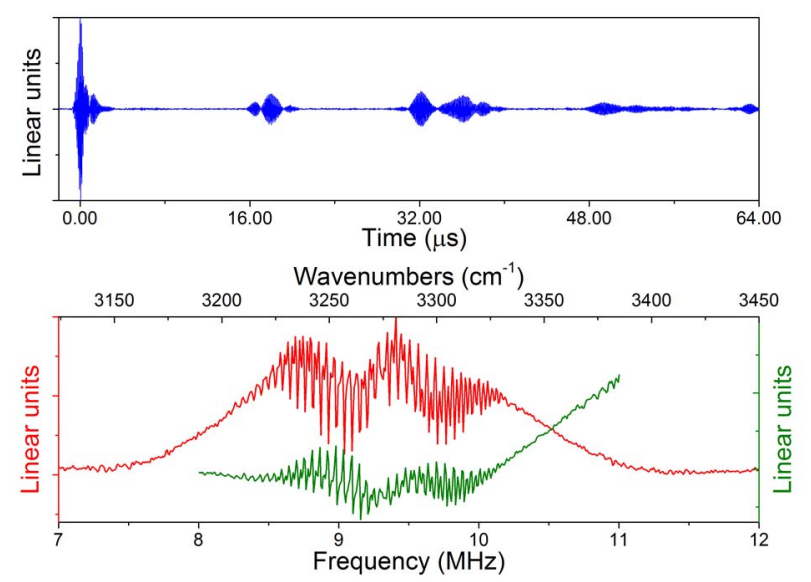

Fig. 5. Time domain interferogram (upper panel) of acetylene $\left(\mathrm{C}_{2} \mathrm{H}_{2}, 10 \%\right.$ in $\mathrm{N}_{2}$, pressure 100 mbar, $30 \mathrm{~cm}$ path length, $\Delta f=46 \mathrm{~Hz}$ ). Lower panel: Power spectrum (red) and dispersion information (green) in the $\mathrm{RF}$ domain obtained by considering the $120 \mu \mathrm{s}$ interferogram. of acetylene $\left(\mathrm{C}_{2} \mathrm{H}_{2}\right)$ at a concentration of $10 \%$ in nitrogen (pressure 100 mbar, $30 \mathrm{~cm}$ path length, $\Delta f=46 \mathrm{~Hz}$ ). On the interferogram, the wave packages after the initial burst indicate that optical frequency components of the comb source are absorbed and dispersed by the gas sample. The FFT of the interferogram recorded within $120 \mu \mathrm{s}$ gives the acetylene absorption spectrum between 3150 and $3400 \mathrm{~cm}^{-1}$ with a $\mathrm{RF}$ resolution of $7.6 \mathrm{kHz}$, corresponding to an infrared spectral resolution of $15 \mathrm{GHz}$ $\left(0.5 \mathrm{~cm}^{-1}\right)$. Figure $6(\mathrm{a})$ shows the first $12 \mu \mathrm{s}$ of the recorded interferogram of methane $\left(\mathrm{CH}_{4}, 1 \%\right.$ in nitrogen, 1 bar pressure, $30 \mathrm{~cm}$ path length, $\Delta f=117 \mathrm{~Hz}$ ). The recorded $60 \mu \mathrm{s}$ interferogram carries the information of a $250 \mathrm{~cm}^{-1}$ wide spectrum between 2950 and $3200 \mathrm{~cm}^{-1}$ with a $\mathrm{RF}$ resolution of $15.2 \mathrm{kHz}$, corresponding to an infrared spectral resolution of $11.7 \mathrm{GHz}\left(0.39 \mathrm{~cm}^{-1}\right)$. In Fig. 6(c), the absorption and dispersion spectra from methane are obtained from a single shot interferogram within $60 \mu \mathrm{s}$ and compared after background removal $[26,27]$ with a simulated spectrum, based on the HITRAN database $\left(0.4 \mathrm{~cm}^{-1}\right.$ resolution). The experimental and simulated spectra agree quite well for the recorded $\mathrm{P}, \mathrm{Q}$ and $\mathrm{R}$ branches. Below $3000 \mathrm{~cm}^{-1}$, distortions are present due to a lower signal-to-noise ratio (SNR) observed in the RF spectrum (below $30 \mathrm{MHz}$ ) enhanced by the background removal calculation [26]. The other spectral distortions are mainly due to the remaining frequency jittering of the laser combs since the offset frequencies of the pumps are free-running [28].

The OPO cavity reported has several advantages for dual-comb spectroscopy. Both generated idlers originate from the same OPO cavity experiencing identical disturbances, such as mirror vibrations and air or temperature fluctuations. The shared OPO cavity cancels out much of the instabilities compared to using two separate OPOs as dual-comb sources. Moreover, the two signal beams resonating in the optical cavity are counterpropagating and
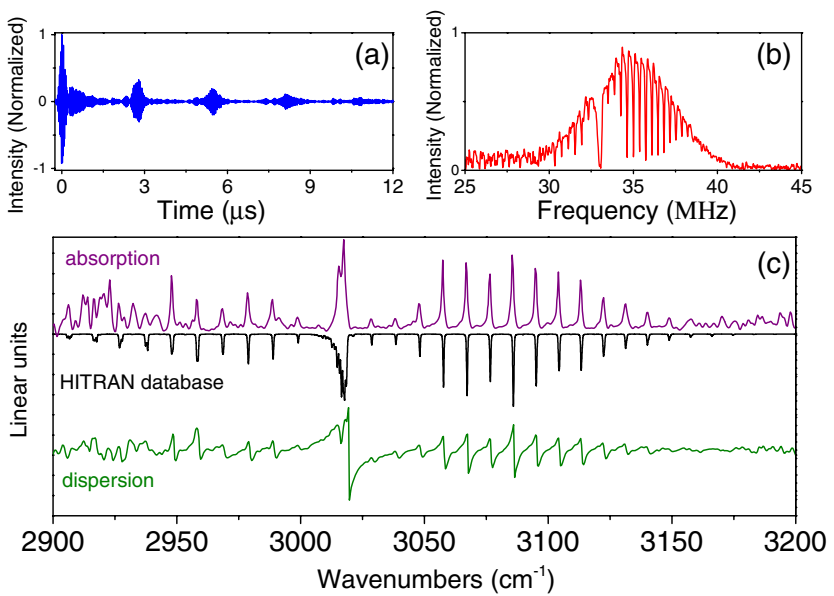

Fig. 6. (a) Time domain interferogram of methane $\left(\mathrm{CH}_{4}\right)$ over $12 \mu \mathrm{s}$. (b) Apodized power spectrum calculated from a $60 \mu \mathrm{s}$ recorded interferogram. (c) Comparison between the measured (purple curve) and simulated (black curve, HITRAN database) absorption spectrum of methane. The $\mathrm{Q}$ branch $\left(\right.$ at $3050 \mathrm{~cm}^{-1}$ ), the $\mathrm{R}$ branch (right side), and the beginning of the $\mathrm{P}$ branch (left side) of the $\nu_{3}$ vibrational transition of methane can be clearly observed. The green curve shows the dispersion information after background removal. 
do not affect the generation of the two idler beams. With this new cavity design, the spectrum overlap of the two idlers can be maximized by independently adjusting the temperature of each crystal. Furthermore, by two independent outputs, dispersion spectra can be measured, which might be beneficial for trace gas sensing [29,30]. For the dual-comb configuration as well as for Fourier spectroscopy, the amplitude of the dispersion signal should be immune to the intensity fluctuations and can provide more reliable and accurate background free data than with the absorption spectrum [26,27]. In our system, the main remaining noise sources are the detector noise and the frequency jittering of the combs (due to the pump fluctuations). They affect the SNR, the spectral resolution, and the efficiency of long-time averaging. A better stabilization of the combs or adaptive sampling techniques [28] will be considered to achieve measurement with higher spectral resolution and higher SNR by multiple spectrum averages.

In summary, we have reported a dual-comb FTS based on a high-power singly resonant OPO with two MgO: PPLN crystals in a ring cavity. This is demonstrated with an absorption and dispersion spectrum of methane covering $250 \mathrm{~cm}^{-1}\left(2950-3200 \mathrm{~cm}^{-1}\right)$ in the mid-infrared wavelength region, recorded within $60 \mu \mathrm{s}$. In addition, the acetylene spectrum is recorded by tuning the OPO to another spectral region covering $250 \mathrm{~cm}^{-1}$ and centered at $3250 \mathrm{~cm}^{-1}$. Since the mid-infrared frequency combs are not yet fully stabilized, these initial results give an overview of the advantages offered by the presented innovative OPO cavity to achieve accurate, high-spectral-resolution, broadband absorption and dispersion spectroscopy for real-time chemical sensing.

This work is financially supported by the Dutch STW project 11830 and the EU en Province Gelderland within the GO-EFRO project: 2009-010034 "Gas analysis systems for quality control of agricultural products and medical diagnostics".

\section{References}

1. I. Coddington, W. C. Swann, and N. R. Newbury, Phys. Rev. Lett. 100, 013902 (2008).

2. A. Schliesser, N. Picque, and T. W. Hansch, Nat. Photonics 6, 440 (2012).

3. K. A. Tillman, R. R. J. Maier, D. T. Reid, and E. D. McNaghten, Appl. Phys. Lett. 85, 3366 (2004).

4. J. H. Sun, B. J. S. Gale, and D. T. Reid, Opt. Lett. 32, 1414 (2007).
5. F. Adler, K. C. Cossel, M. J. Thorpe, I. Hartl, M. E. Fermann, and J. Ye, Opt. Lett. 34, 1330 (2009).

6. Z. Zhang, X. Fang, T. Gardiner, and D. T. Reid, Opt. Lett. 38, 2077 (2013).

7. N. Leindecker, A. Marandi, R. L. Byer, and K. L. Vodopyanov, Opt. Express 19, 6296 (2011).

8. N. Leindecker, A. Marandi, R. L. Byer, K. L. Vodopyanov, J. Jiang, I. Hartl, M. Fermann, and P. G. Schunemann, Opt. Express 20, 7046 (2012).

9. M. J. Thorpe and J. Ye, Appl. Phys. B 91, 397 (2008).

10. S. A. Diddams, L. Hollberg, and V. Mbele, Nature 445, 627 (2007).

11. C. Gohle, B. Stein, A. Schliesser, T. Udem, and T. W. Hänsch, Phys. Rev. Lett. 99, 263902 (2007).

12. F. Adler, P. Masłowski, A. Foltynowicz, K. C. Cossel, T. C. Briles, I. Hartl, and J. Ye, Opt. Express 18, 21861 (2010).

13. J. Mandon, G. Guelachvili, and N. Picque, Nat. Photonics 3, 99 (2009).

14. F. Keilmann, C. Gohle, and R. Holzwarth, Opt. Lett. 29, 1542 (2004).

15. A. Schliesser, M. Brehm, F. Keilmann, and D. van der Weide, Opt. Express 13, 9029 (2005).

16. E. Baumann, F. R. Giorgetta, W. C. Swann, A. M. Zolot, I. Coddington, and N. R. Newbury, Phys. Rev. A 84, 062513 (2011).

17. T. Ideguchi, A. Poisson, G. Guelachvili, T. W. Hänsch, and N. Picqué, Opt. Lett. 37, 4847 (2012).

18. Z. Zhang, T. Gardiner, and D. T. Reid, Opt. Lett. 38, 3148 (2013).

19. B. Bernhardt, A. Ozawa, P. Jacquet, M. Jacquey, Y. Kobayashi, T. Udem, R. Holzwarth, G. Guelachvili, T. W. Hansch, and N. Picque, Nat. Photonics 4, 55 (2009).

20. M. J. Thorpe, K. D. Moll, R. J. Jones, B. Safdi, and J. Ye, Science 311, 1595 (2006).

21. M. J. Thorpe, D. Balslev-Clausen, M. S. Kirchner, and J. Ye, Opt. Express 16, 2387 (2008).

22. S. Schiller, Opt. Lett. 27, 766 (2002).

23. Y. Wang, M. G. Soskind, W. Wang, and G. Wysocki, Appl. Phys. Lett. 104, 031114 (2014).

24. G. K. Samanta, A. Aadhi, and M. Ebrahim-Zadeh, Opt. Express 21, 9520 (2013).

25. P. Vladimir, Laser Heterodyning (Springer, 2009).

26. J. A. de Haseth and P. R. Griffiths, Fourier Transform Infrared Spectrometry (Wiley, 2007).

27. J. Kauppinen and J. Partanen, Fourier Transforms in Spectroscopy (Wiley, 2001).

28. T. Ideguchi, A. Poisson, G. Guelachvili, N. Picqué, and T. W. Hänsch, Nat. Commun. 5, 3375 (2014).

29. G. Wysocki and D. Weidmann, Opt. Express 18, 26123 (2010).

30. M. Nikodem and G. Wysocki, Ann. N.Y. Acad. Sci. 1260, 101 (2012). 